\title{
Programa educativo de técnicas y hábitos de estudio para lograr aprendizajes sustentables en estudiantes de nuevo ingreso al nivel superior
}

\section{Educational program focused on the techniques and study habits to achieve sustainable learning in new students at the upper level}

\author{
Ramón Bedolla Solano \\ Universidad Autónoma de Guerrero (UAGRO), México
}

\begin{abstract}
Resumen
Reflexionar sobre los hábitos que deben formar y las técnicas de estudio que deben aplicar estudiantes para obtener aprendizajes sustentables, contribuye en perfeccionar su rendimiento académico en la universidad. Se realizó un estudio mixto entre junio-agosto de 2017, se enfocó en el análisis de un Programa Educativo de Técnicas y Hábitos de Estudio implementado a 62 estudiantes de nuevo ingreso a un programa de estudio universitario, contempló tres etapas, diseño, aplicación y evaluación. Desde la primera fase y hasta la última, se evaluaron conocimientos previos y aprendizajes, por lo cual, en algunas sesiones, se aplicaron instrumentos como el test y la entrevista. Los resultados evidenciaron que al principio, la mayoría de los jóvenes contaban con conocimientos superficiales sobre las técnicas y hábitos de estudio, con la evaluación realizada durante el desarrollo del programa se reforzó la adquisición de aprendizajes sobre estas temáticas; asimismo, al realizar una evaluación general sobre la eficiencia del programa, se pudo percibir que implementar programas educativos de esta naturaleza en estudiantes de nuevo ingreso a la universidad posibilita la manera de fomentar estrategias para adquirir aprendizajes sustentables.
\end{abstract}

Palabras claves: Técnicas de estudio; hábitos de estudio; competencias; aprendizaje sustentable.

\begin{abstract}
Reflect on the habits that must be formed and the study techniques that students must apply to obtain sustainable learning contributes to improve their academic performance in the university. A study with the mixed approach was carried out between June-August 2017, focused on the analysis of an Educational Program of Techniques and Study Habits implemented to 62 new students to a university study program; it contemplated three stages, design, application and evaluation. From the first phase to the last one, previous knowledge and learning were evaluated, for which, in some sessions, instruments such as the test and the interview were applied. The results showed that at the beginning, most of the young people had superficial knowledge about the techniques and study habits, with the evaluation made during the development of the program the acquisition of learning about these topics was reinforced in the majority, likewise, when carrying out a general evaluation on the efficiency of the program, it could be perceived that implementing educational programs of this nature in newly admitted students to the university makes possible the ways of promoting strategies to acquire sustainable learning or that can serve in present or future situations.
\end{abstract}

Keywords: Study techniques; study habits; competences; sustainable learning. 


\section{Introducción}

Investigar sobre los hábitos y técnicas de estudio en el campo educativo es sumamente importante por los hallazgos encontrados, para identificar aquello que afecta o beneficia el rendimiento académico y algún otro suceso estudiantil, en consecuencia, se aplican métodos científicos que llevan a determinar propuestas o acciones que pueden atender esta situación. Estudios realizados sobre esta temática, han concluido en la falta de implementación de hábitos y técnicas de estudios y esto repercute en bajos rendimientos académicos y, por ende, otros problemas que se asocian a ello.

La importancia de que los estudiantes apliquen estas técnicas, incide en obtener una visión global o específica de lo que se desea aprender aplicando diversas herramientas que le faciliten la comprensión, mientras que los hábitos, son esas actitudes que deben de poner en práctica de manera cotidiana y que deben de promover y formar para perfeccionar su rendimiento académico en la universidad. Los términos que se resaltan en estas líneas conllevan a realizar un análisis para comprenderlos, debido a que existen algunos detalles que pueden llevar a la confusión. Un ejemplo, la manera en que se percibe y se entiende o comprende lo que es estudiar con leer. Muchas personas comprenden de la misma manera estudiar y leer. Arguelles (2014) manifiesta que no es lo mismo estudiar con leer, sin embargo, cita a Garrido para explicar esta aseveración, él explica que "estudio es una actividad obligatoria, cuyo propósito es memorizar cierta información, por el tiempo necesario para cubrir el trámite de examen y que generalmente incluye una simulación de la lectura. Entiendo por lectura una actividad voluntaria, cuyo propósito es comprender, dar significado al texto por el gusto de hacerlo, pues sin comprensión no hay forma de mantener vivo el interés". El señalamiento anterior automáticamente hace saber, que el estudiar es un proceso más a fondo y que el leer es una herramienta para llevar a la comprensión. En este tenor, a veces suele decirse "voy a leer y/o estudiar un libro" y entonces, se debe tener en cuenta que el estudiar es algo más complicado porque para llegar a la comprensión y al análisis tienes que emplear varias estrategias.

Implementar las técnicas y fomentar los hábitos de estudio en los estudiantes resultaría de suma importancia en las instituciones educativas principalmente en las universidades. Se necesita, y es urgente atender. La falta de estas técnicas conlleva a resultados no satisfactorios en los procesos de aprendizajes de algunos estudiantes, es de gran interés que se conozcan indispensablemente y a que se refieren para que las implementen. En este orden de ideas, los profesores tienen que 
considerar las competencias en este campo porque de algún modo u otro, tienen que promoverlas para que consigan que sus estudiantes gestionen aprendizajes sustentables y un buen desempeño.

Este artículo expone información acerca de las técnicas y hábitos de estudios, de un programa que obedeció a estás temáticas, se implementó a estudiantes de nuevo ingreso al Programa Educativo de Sociología de la Comunicación y Educación, de la Universidad Autónoma de Guerrero, UAGro de la generación 2017-2021. Este curso fue organizado y planificado. Sus etapas se conciben en su diseño, implementación y evaluación y se sustentó en el constructivismo, competencias, aprendizaje significativo y sustentable.

La investigación, se desarrolló entre los meses de junio a agosto de 2017. En el mes de junio y julio se diseñó el programa, durante este lapso, se aplicó un test, con variables (técnicas y hábitos de estudio) para identificar conocimientos sobre esta temática, en agosto, la segunda semana, se implementó y se realizó una primera evaluación, a finales del mes mencionado, se concluyó la etapa de evaluación y se obtuvieron los resultados. Este curso se enfocó en analizar la eficiencia del Programa Educativo de Técnicas y hábitos que se aplicó a estudiantes de nuevo ingreso del ciclo escolar 2017-2021, estuvo constituido en su diseño, implementación y su evaluación. Fue un estudio mixto. Con respecto a los resultados y atendiendo a como se constituyó este programa, se tuvo como producto, en la parte de diseño, un programa educativo del tema en cuestión y su secuencia didáctica fundamentados en los supuestos constructivistas y de competencias, también se logró identificar los hábitos y técnicas que estudiantes adquirieron en niveles anteriores y en lo que respecta a su implementación, este programa implementó a un total de 62 estudiantes solicitantes al programa educativo de Sociología de la Comunicación y Educación de la Universidad Autónoma de Guerrero en Acapulco, México. Durante el desarrollo de las sesiones se realizó una evaluación mixta sobre aprendizajes adquiridos, La investigación logró sus propósitos. Los estudiantes lograron la comprensión de la importancia de formar hábitos y aplicar técnicas de esta índole en sus estudios.

\section{Antecedentes del estudio}

Los alumnos que entran a estudiar en un nivel superior se ven desubicados en sus estudios debido al exceso de información y al poco tiempo disponible (Sepúlveda y Lucia, 2017). El uso de técnicas de estudio es imprescindible, no solo para algunos, sino para todos los alumnos. La falta de uso de estas herramientas, imposibilitan 
a muchos estudiantes a lograr sus propósitos, es decir, aprender. Marcelo (2011) en una investigación descriptivo-correlacional de la UAB, donde seleccionó estudiantes de cinco facultades permitió relacionar las técnicas y hábitos de estudio con el rendimiento académico y dedujo que los estudiantes utilizan las técnicas de estudio; sin embargo, aquellos con rendimiento sobresaliente lo hacen de manera más efectiva. En relación con los hábitos de estudio, dedujo que los hábitos adquiridos en la familia, y sus primeros años de formación repercuten significativamente en el rendimiento.

\section{La actividad del estudio}

Estudiar es un proceso muy complicado, implica implementar un conjunto de estrategias y técnicas para llegar al conocimiento o la adquisición de competencias. Un estudiante ha de comprender aquello que estudia, iniciando su camino más allá de la memorización y es por ello que pensamos es tan importante el uso de recursos o estrategias (Guirao, 2013). Según la Real Academia de la Lengua Española (2013: Sepúlveda y Lucia, 2017b) estudiar es "ejercitar el entendimiento para alcanzar o comprender algo". Por lo tanto, estudiar es saber aprender, es saber aplicar las capacidades cognitivas y motoras a la adquisición, comprensión y organización de conocimientos (op. cit.). "Según los expertos y la propia UNESCO son cuatro los grandes tipos de competencias que los sujetos tienen que adquirir para garantizar que han tenido una formación integral (Delors, 1996). Ellas son aprender a conocer, Aprender a hacer, aprender a vivir juntos y aprender a ser" (Modelo Educativo UAGro, 2013). La función de la escuela ya no es enseñar a los niños y jóvenes lo que no saben, sino aquello que necesitan para aprender a aprender. El currículo nacional debe fomentar el desarrollo de competencias para la vida que son fundamentales. Una competencia clave que estructura a otras es "aprender a aprender", que significa aprender a pensar, a cuestionarse acerca de los diversos fenómenos, sus causas y consecuencias, etc. (SEP, Modelo Educativo, 2016).

\section{Diferenciación entre la técnica y la estrategia}

Para abordar estos términos es conveniente comprender lo que es método, debido a que en el campo educativo, se comprende igual en algunos casos. El concepto de método también ha sido muy utilizado en el ámbito pedagógico con ese mismo nombre, o bien con el nombre equivalente de estrategia didáctica (Gimeno, 1986: Elizondo, 2000). De manera general y en otros campos, el método es un procedimiento que implica cierto orden en el desarrollo de sus acciones para 
llegar a la meta. Sin embargo, dentro de este conjunto de acciones, podemos encontrar que las estrategias son necesarias, y es importante dejar claro que estas son flexibles, no obstante, son parte de un método tal vez rígido porque tiene sustento en ciertos paradigmas. En sí, para que las estrategias se lleven a cabo, son importante, las técnicas, Una estrategia emplea diversas técnicas para conseguir lo que se propone, la técnica se limita, la estrategia es amplia, abarca un todo. Para profundizar un poco más, se describe lo siguiente "es importante diferenciar la técnica de la estrategia, pues como afirman Pozo y Gómez (2001:59-63), aunque ambos son procedimientos, difieren en cuanto a las situaciones que los incentivan y en las que se ponen en práctica. De un lado, la técnica se caracteriza por ser una actividad rutinaria, repetitiva y mecánica, en fin, una técnica es un procedimiento para resolver los famosos ejercicios. La estrategia tiene que ver con una actividad planificada, que se pone en juego para resolver un problema, es decir, en una tarea novedosa" (Castro, 2005).

\section{Las técnicas en el campo educativo}

Dentro del campo educativo, las técnicas se consideran procedimientos, están implícitas en las estrategias. Se pide a estudiantes implementar técnicas para lograr comprender algo que es de interés para su formación, en lo académico y lo cotidiano. Enseñamos una técnica cuando queremos que nuestros alumnos y alumnas conozcan y utilicen un procedimiento para favorecer la tarea del estudio (Barrena y Molina, 2010). De acuerdo a DefinicionABC Tu diccionario hecho fácil, las técnicas de estudio son esas herramientas que puede utilizar un alumno para facilitar el aprendizaje en la comprensión de un texto. Hay una serie de técnicas que ayudan a mejorar y a rentabilizar el estudio; no son, en modo alguno, infalibles, ni tampoco imprescindibles para todos. Cada persona deberá, en todo caso, primeramente, conocerlas, y, después, elegir aquellas que mejor se adapten a su forma de aprender y retener los contenidos, e, incluso, «adaptarlas» o «inventar» nuevas maneras de llevarlas a la práctica (Sebastián, Ballesteros y Sánchez, 2012). Cuando se habla de las técnicas de estudio uno asocia que están dirigidas a un determinado grupo de alumnos con intención de mejorar su proceso de estudio. Esta aplicación es cierta, y quizá, la más extendida, pero como docentes también nos interesa conocer las técnicas de estudio para aplicarlas en nuestra propia asignatura (Valero, 2011). Las técnicas de estudio son un conjunto de herramientas, fundamentalmente lógicas, que ayudan a mejorar el rendimiento y ayudan el proceso de memorización y 
estudio. Estudiar es situarse adecuadamente ante unos contenidos, interpretarlos, asimilarlos, retenerlos, para después poder expresarlos en una situación de examen o utilizarlos en la vida práctica (Publicaciones Vértice S.L., 2008).

\section{Los hábitos de estudio}

Los hábitos de estudio han sido investigados a partir de distintas corrientes teórico metodológicas, intentando demostrar su eficacia en el desarrollo académico de los estudiantes en todos los niveles educativos (Mira y López, 1995: Enríquez, 2013). Los hábitos son conductas o comportamientos que son repetidos por las personas continuamente; sin embargo, no son innatos, es decir, no se nace con ellos, sino que se van formando (SEP, 2014a). Los hábitos de estudio son estrategias, técnicas, recursos, actitudes y actividades que facilitan la adquisición de conocimientos, es decir, permiten desarrollar o mejorar el rendimiento académico (SEP, 2014b). Los hábitos de estudio han sido considerados elementos imprescindibles durante la formación académica, al asumir que los estudiantes universitarios tienen y ejecutan estrategias para el estudio que les facilitan su desarrollo académico; sin embargo, son muchos los casos en los que se presentan dificultades y esto puede abrir las puertas al ausentismo o, incluso, la deserción (Castillo, 2015). Los componentes de los hábitos de estudio se pueden agrupar en cuatro grandes rubros: 1) estrategias de higiene: se establecen las condiciones que permiten mantener y propiciar la salud física y mental; 2) condiciones de los materiales: se consideran en su totalidad los recursos y materiales necesarios para ejercer el estudio; 3) estrategias de estudio: corresponde a las formas de estudiar para adquirir el conocimiento, y 4) capacidades de estudio referentes a las acciones mentales que se poseen y se dominan para mejorar el aprendizaje (Escalante, 2005: Castillo, 2015). La preocupación de los hábitos de estudio de los alumnos viene de tiempo atrás, no obstante, en nuestro tiempo esta cuestión ha adquirido un renovado interés por la extensión de la educación y por las altas tasas de fracaso escolar (Cambrón, Chávez y Llamas, 2016).

\section{El rendimiento escolar como producto de la implementación de técnicas y hábitos de estudio}

El rendimiento escolar en un primer acercamiento se puede concebir como el grado de conocimientos que posee un estudiante de un determinado nivel educativo a través de la escuela. La forma como una institución educativa expresa ese grado cognitivo se refleja en la calificación escolar, la cual le es asignada al alumno por el profesor. Como es conocido, en el plantel escolar las diferencias de rendimiento 
entre los individuos son expresadas en términos de una escala, en su mayoría numérica, cuyos extremos indican el más alto y el más bajo rendimiento (Gutiérrez y Montañez, 2012). El rendimiento escolar es un "nivel de conocimientos demostrado en un área o materia comparado con la norma de edad y nivel académico" (Jiménez en Edel, 2003). Carpio (1975) define rendimiento académico como el proceso técnico pedagógico que juzga los logros de acuerdo a objetivos de aprendizaje previstos (Guerrero, 2010).

\section{Enfoques del programa de técnicas y hábitos de estudio}

El programa de técnicas y hábitos de estudio implementado se enmarcó en un enfoque constructivista, de competencias, aprendizajes significativos y aprendizaje sustentable. De acuerdo a (Tobón 2008: Casaña, 2015) las competencias no son más que: "Procesos complejos de desempeño con idoneidad en determinados contextos, integrando diferentes saberes (saber ser, saber hacer, saber conocer y saber convivir)". En el construccionismo social la realidad aparece como una construcción humana que informa acerca de las relaciones entre los individuos y el contexto y el individuo aparece como un producto social -el homo socius-, definido por las sedimentaciones del conocimiento que forman la huella de su biografía, ambiente y experiencia (Serrano y Pons, 2011).El aprendizaje significativo es una teoría que se ocupa del proceso de construcción de significados por parte de quien aprende, que se constituye como el eje esencial de la enseñanza (Rodríguez, 2014). El aprendizaje sustentable, es aquél en el que la información recibida o parte de ella fue apropiada correctamente como nuevo conocimiento, aumentando y enriqueciendo la estructura cognitiva previamente existente (Galagovsky, 2004). El término sustentable se aprecia con más detalles en el campo ambiental por el hecho de buscar un equilibrio entre el medio y los recursos, sin embargo, cabe aclarar que se puede aplicar en otros campos, como en este caso al educativo. Algo es sustentable cuando hay un equilibrio, cuando se satisfacen las necesidades actuales y no se afectan las del futuro. En el campo educativo, una educación es sustentable cuando es de calidad y por ende, en el terreno de los aprendizajes, estos son sostenibles, si en el proceso de su adquisición se aplicaron las técnicas y se formaron los hábitos adecuados. Los aprendizajes sustentables resultan ser fundamentales en los alumnos por el hecho de organizar, seleccionar e integrar información útil para situaciones presentes o futuras. 


\section{Objetivo}

1. El propósito principal de esta investigación se enfocó en generar hábitos y técnicas de estudio, a través de un programa educativo fundamentado en supuestos constructivistas para coadyuvar en aprendizajes sustentables en estudiantes de nuevo ingreso a la universidad.

Por consiguiente y para cumplir con ello:

\section{Objetivo específicos}

1. Se identificó a través un test en la etapa de diseño de dicho programa los conocimientos sobre hábitos y técnicas de estudio en los estudiantes muestra de este estudio.

2. Se diseñó y elaboró un Programa Educativo de Técnicas y Hábitos de Estudios con un enfoque constructivista, por competencias y aprendizaje sustentable orientado a estudiantes de nuevo ingreso al Programa Educativo de Sociología de la Comunicación y Educación, generación 2017-2021.

3. Se aplicó el programa educativo en cuestión para fomentar hábitos y generar técnicas de estudios que permitan a los estudiantes adquirir aprendizajes sustentables y competencias significativas y así perfeccionar su rendimiento académico en la escuela en situaciones presentes y futuras.

4. Se evaluó cualitativa y cuantitativamente los aprendizajes adquiridos, así como también, la eficacia del programa educativo.

\section{Metodología a desarrollar}

La investigación es mixta debido a que emplea técnicas cuantitativas y cualitativas, finalmente se concluye en una descripción de los hechos encontrados. La investigación se realizó durante los meses de junio-agosto de 2017. Este trabajo se desarrolló en tres etapas: Durante los meses de junio-julio, se trabajó en el diseño del programa educativo sobre las técnicas y hábitos de estudio. Implementación, durante los días, 14 al 18 de agosto, se implementó el programa de técnicas de estudio y la evaluación, se realizó antes del diseño, durante la puesta en marcha del programa y hasta los últimos días del mes de agosto del año en cuestión. 


\section{Población}

El presente estudio es producto de la implementación de un Programa Educativo sobre Técnicas y Hábitos de Estudio, que se orientó a estudiantes de nuevo ingreso (ciclo 2017-2021) a la Escuela Superior de Sociología que ofrece el programa sociología de la comunicación y educación y depende de la Universidad Autónoma de Guerrero (UAGro), ubicada en Acapulco, México del ciclo escolar 2017-2021. El anuario estadístico 2014-2015 de la UAGro, establece que la escuela de Sociología cuenta con una planta docente de 15 docentes y 287 estudiantes. Para el periodo 2017-2021, se registraron alrededor de 80 estudiantes, y asistieron a tomar el curso un total de 62.

\section{Muestra}

Se hace hincapié que la investigación es mixta, por esta razón y por así considerarlo, la obtención de la muestra, se debió al enfoque cualitativo, un procedimiento estadístico para su obtención no se realizó. En el enfoque cualitativo, se establece recolectar datos, sin que necesariamente sea representativo del universo o población que se estudia (Hernández et al, 2007; Angulo, 2011). Se eligió a todos los estudiantes, sin embargo, se trabajó con 62 (42 mujeres y 20 hombres). De acuerdo a la organización e indicaciones en la escuela, estas personas tenían que estar agrupadas en el grupo " $A$ " $y$ " $B$ ". Por consiguiente, el primero, estuvo constituido de 28 jóvenes (19 mujeres y 9 hombres) y el segundo de 34 (23 mujeres y 11 hombres).

\section{Técnicas empleadas para la recolección de información}

Primera fase: El diseño del Programa de Técnicas y hábitos de Estudio. Durante los meses de junio-julio, se trabajó en el diseño del programa y su respectiva secuencia didáctica donde se enlistan y planifican las actividades específicas, como lo son temas a ser vistos en las diferentes sesiones, las estrategias pedagógicas-didácticas a ser implementadas por el docente, la forma de evaluar, los aprendizajes y competencias que formaran los estudiantes, en esta etapa, se aplicó un test para identificar los conocimientos sobre los hábitos y técnicas de estudios.

Segunda fase: La implementación del Programa de Técnicas y Hábitos de Estudio. La puesta en marcha del curso en cuestión, se dio del 14 al 18 de agosto del 2017. Las actividades del curso en cuestión tuvieron su sustento en el programa y la secuencia. Durante las cinco sesiones que comprendió el programa se reali- 
zaron actividades, ejercicios, etc. que tenían que ver con las técnicas y hábitos de estudio, estas se fueron registrando y a la vez evaluando para identificar el nivel de comprensión y a la vez los aprendizajes.

Tercera fase: La evaluación del Programa de Técnicas de Estudios. La evaluación, se realizó en todo el proceso, es decir, desde el inicio hasta que se concluyó el programa. En la última sesión se aplicó una encuesta con el objetivo de comparar lo que contestaron en la primera aplicación (en el diseño) y después (última clase) para analizar las diferencias en conocimientos y aprendizajes adquiridos. Al finalizar el curso, se realizaron algunas preguntas a los estudiantes a cerca de algunos aspectos del curso, como, por ejemplo, papel del facilitador, recursos de aprendizajes, aprendizajes adquiridos, etc. esto con la finalidad de conocer la eficiencia del programa.

\section{Procesamiento de la información}

Con respecto al diseño del programa, se tuvo que recabar información valiosa que fundamentara este programa. Se recurrió a identificar los enfoques teóricos didáctico-pedagógicos (competencia, constructivismo, aprendizaje significativo y el fundamento de un aprendizaje sustentable). Se identificaron los formatos y elementos para construir el programa y la secuencia didáctica. La información fue analizada y plasmada en los formatos dichos para construir el programa y la secuencia didáctica de este curso taller. La información recabada durante la puesta en marcha de este programa cuyo sustento se plasmaba en el programa y la secuencia, fue procesada cualitativamente, es decir, las actividades, la entrega de evidencias, las tareas, etc. que realizaban los estudiantes en cada sesión fueron revisadas y analizadas para identificar los aprendizajes y competencias adquiridas. En relación a los instrumentos aplicados, estos se procesaron de manera cuantitativa y cualitativa, es decir, se organizó la información de manera cuantitativa y en consecuencia se analizaron cualitativamente los resultados. Finalmente, estos se plasmaron en la descripción de los sucesos encontrados por el programa desarrollado.

\section{Resultados}

El programa educativo de este curso fue plasmado en un formato para elaborar programas de unidades de aprendizajes o materias de nivel licenciatura en la UAGro. Se consideró este formato, por la razón de que es el oficial de esta institución, 
además, contempla una estructura ideal ya que integra los elementos necesarios para diseñar y elaborar un programa de curso basado en enfoques constructivistas, de aprendizajes significativos, sustentables y de competencias.

Lo que se contempló en el formato para elaborar el programa fue la información general como información de la institución donde se implementaría el curso, la modalidad, en este caso fue un curso presencial, el periodo, una semana, se identificaron y se registraron las competencias previas requeridas, para ello, se solicitaron las estrategias de estudios y hábitos adquiridos en su nivel de educación anterior, el número de horas con el facilitador y de manera independiente que se trabajarían en la semana o periodo que consideró este taller. Fueron alrededor de diez horas de trabajo con el facilitador y cinco de tareas o de trabajo independiente. Contribución del curso en su formación y la competencia a adquirir: Se redactaron algunos elementos que contempla el programa, como la manera en que los aprendizajes contribuyen, por esta razón, se registró que ellos se reflejarían en cambio de actitudes y métodos para organizar y comprender la información.

\section{La elaboración de la secuencia didáctica del curso, contempló los siguientes elementos}

En la secuencia o planeación didáctica, se registraron cinco sesiones, en cada sesión, de acuerdo a lo que contempló el diseño de este formato, se registró también, la fecha de cada una de las sesiones, las actividades de apertura, desarrollo y cierre que se tenían que realizar entre los estudiantes y el profesor. Se registraron las tareas que se tenían que hacer por cada sesión, así como también, los aprendizajes que debían de adquirir los estudiantes por cada clase. En esta misma vertiente, con respecto a la evaluación, se consideraron las evidencias que debían de presentar por actividades presenciales e independientes, aunado a ello, la ponderación, es decir, el valor que debía tener cada sesión, de tal manera que al final, las cinco sesiones dieran un $100 \%$.

\section{Primera evaluación (Evaluación diagnóstica). Evaluación para identificar conocimientos sobre técnicas y hábitos de estudio}

Se aplicó un test para identificar conocimientos sobre hábitos y técnicas de estudio, de 62 estudiantes 45 contestaron el instrumento, 25 del grupo " $A$ " y 20 del grupo "B". En el grupo "A" fueron 18 mujeres y 7 hombres, en el grupo " $B$ ", fueron 16 mujeres y cuatro hombres. 
La mayoría de los estudiantes son egresados de bachilleratos universitarios, de la UAGro y el resto de instituciones técnicas que hay en Acapulco, en gran medida de las instituciones de procedencia son públicas, uno o dos estudiantes fueron egresados de escuelas privadas.

El instrumento aplicado tuvo su fundamento en el test para la evaluación de hábitos de estudios del Colegio Peter College (su fuente aparece en referencias), sin embargo, este sufrió algunos cambios y adaptaciones para ser aplicado con los estudiantes de nuevo ingreso de la institución universo de este estudio. Las variables consideradas fueron similares a la del instrumento de dicho colegio, estas fueron, actitudes ante el estudio en el hogar, actitudes ante el estudio durante la realización de su clase, actitudes ante el estudio después de la escuela, actitudes generales ante el estudio y técnicas de estudio (esta variable fue adaptada al test). Fueron 50 preguntas, cada variable tenía incluida de 6 a 10 preguntas. Cabe aclarar que las preguntas que consideraba el instrumento fueron modificadas, en el sentido de identificar el nivel de conocimiento que tienen los estudiantes de nuevo ingreso sobre las técnicas y hábitos de estudio. Los estudiantes evaluaron cada una de las preguntas con las respuestas nunca (1), casi nunca (2), algunas veces (3), casi siempre (4) y siempre (5). Las respuestas obtenidas se agruparon en la siguiente tabla: Información del grupo "A":

\section{TABLA I}

Diagnóstico del nivel de conocimientos de técnicas y hábitos de estudios de estudiantes de nuevo ingreso (Grupo A).

\begin{tabular}{|l|c|c|c|c|c|}
\hline \multicolumn{1}{|c|}{ Variable } & 1 & 2 & 3 & 4 & 5 \\
\cline { 2 - 6 } & Nunca & $\begin{array}{c}\text { Casi } \\
\text { nunca }\end{array}$ & $\begin{array}{c}\text { Algunas } \\
\text { veces }\end{array}$ & $\begin{array}{c}\text { Casi } \\
\text { siempre }\end{array}$ & Siempre \\
\hline A.- Actitudes ante el Estudio en el Hogar & $2 \%$ & $3 \%$ & $10 \%$ & $15 \%$ & $70 \%$ \\
\hline $\begin{array}{l}\text { B.- Actitudes ante el Estudio durante la } \\
\text { Realización de la Clase }\end{array}$ & $2 \%$ & $3 \%$ & $5 \%$ & $15 \%$ & $75 \%$ \\
\hline C.- Actitudes ante el Estudio después de la Escuela & $1 \%$ & $4 \%$ & $10 \%$ & $15 \%$ & $70 \%$ \\
\hline D.- Actitudes Generales ante el Estudio & $1 \%$ & $3 \%$ & $10 \%$ & $16 \%$ & $70 \%$ \\
\hline E.- Técnicas de estudio & $1 \%$ & $1 \%$ & $8 \%$ & $10 \%$ & $80 \%$ \\
\hline
\end{tabular}

Los resultados que se muestran en la tabla anterior (Grupo A) reflejan que la mayoría de los estudiantes ubicaron sus respuestas en la categoría 4 y 5, y se comprende de manera general que los estudiantes tienen conocimientos sobre los hábitos y técnicas de estudio. Algunos ubicaron sus respuestas en la categoría 3 y pocos en la categoría 2 y 1 . Esto, fue un buen indicador, debido a que indicó que los estudiantes de nuevo ingreso contaban con los conocimientos sobre hábitos y técnicas de estudio. 
Los resultados mostrados en el grupo " $B$ " sobre los conocimientos que los estudiantes tienen sobre los hábitos y técnicas de estudio son similares a los encontrados en el grupo "A". De igual manera fue un buen indicador, los estudiantes, ya tenían conocimientos previos sobre los hábitos y técnicas.

\section{TABLA II}

Diagnóstico del nivel de conocimientos de técnicas y hábitos de estudios de estudiantes de nuevo ingreso (Grupo B).

\begin{tabular}{|l|c|c|c|c|c|}
\hline \multicolumn{1}{|c|}{ Variable } & 1 & 2 & 3 & 4 & 5 \\
\cline { 2 - 6 } & Nunca & $\begin{array}{c}\text { Casi } \\
\text { nunca }\end{array}$ & $\begin{array}{c}\text { Algunas } \\
\text { veces }\end{array}$ & $\begin{array}{c}\text { Casi } \\
\text { siempre }\end{array}$ & Siempre \\
\hline A.- Actitudes ante el Estudio en el Hogar & $2 \%$ & $3 \%$ & $9 \%$ & $15 \%$ & $71 \%$ \\
\hline $\begin{array}{l}\text { B.- Actitudes ante el Estudio durante la } \\
\text { Realización de la Clase }\end{array}$ & $3 \%$ & $2 \%$ & $5 \%$ & $10 \%$ & $80 \%$ \\
\hline C.- Actitudes ante el Estudio después de la Escuela & $2 \%$ & $2 \%$ & $12 \%$ & $14 \%$ & $70 \%$ \\
\hline D.- Actitudes Generales ante el Estudio & $2 \%$ & $3 \%$ & $10 \%$ & $15 \%$ & $70 \%$ \\
\hline E.- Técnicas de estudio & $1 \%$ & $1 \%$ & $8 \%$ & $10 \%$ & $80 \%$ \\
\hline
\end{tabular}

\section{Implementación del Programa de Técnicas y Hábitos de Estudio}

La planeación general y especifica se constituyó en un programa y una secuencia, con estructuras bien definidas que permitieron organizar las actividades de tal manera que se condujera en un orden para cumplir con los propósitos o competencias planteadas. El programa fue implementado del 14 al 18 de agosto del año en curso, 62 estudiantes agrupados en dos grupos dieron seguimiento, sin embargo, algunos jóvenes no asistieron a algunas clases. Fueron 5 sesiones, cada una tuvo una duración de dos horas y por cada sesión se dejó una hora de tarea. Durante estas cinco clases, se realizaron diversas actividades que tenían que ver con las técnicas y hábitos de estudio, estas se fueron registrando y a la vez evaluando para identificar el nivel de comprensión y a la vez los aprendizajes:

Sesión 1, 14 de agosto. Duración 2 horas. Encuadre y presentación del curso. El elemento de competencia fue que los estudiantes comprendieran la metodología y competencia del curso. Las actividades desarrolladas fueron, en este apartado, se presentó a través de power point el programa y la secuencia didáctica, también, lo que concierne a técnicas y hábitos de estudio. Se aplicó la técnica de lluvias de ideas para identificar información previa sobre las técnicas y hábitos de estudio. Se observaron videos sobre estas temáticas. En plenarias se realizaron comentarios. 
De tarea y en un tiempo de una hora, se dejó revisar la técnica del subrayado y aplicarla en una lectura sugerida. Los recursos de aprendizaje se entregaron de manera impresa y digital y estaban contemplados en la secuencia.

Sesión 2, 15 de agosto. Duración 2 horas. Elemento de competencia, comprensión de la técnica del subrayado y del esquema. En esta segunda sesión se recibió la tarea sobre la técnica del subrayado que aplicaron en una lectura sugerida. Los estudiantes observaron videos y una lectura sobre el esquema como técnica para comprender textos. Los estudiantes en equipos elaboraron un esquema para comprender una lectura que trató sobre la Educación Ambiental. Por así considerarlo, los estudiantes continuaron elaborando el esquema y ello fue considerado como trabajo independiente en un tiempo de una hora en la siguiente sesión. Los recursos de aprendizaje se entregaron de manera impresa y digital y estaban contemplados en la secuencia.

Sesión 3, 16 de agosto. Duración 2 horas. El elemento de competencia, fue, Comprensión de la técnica del esquema y del cuadro sinóptico. En esta sesión los equipos expusieron sus esquemas con base en la lectura "Educación Ambiental". Siguiendo la misma dinámica de la sesión anterior, los equipos ya integrados, observaron videos y realizaron lecturas sobre la elaboración de cuadros sinópticos, y se les solicitó hacer un cuadro de este tipo con la misma lectura. Los estudiantes expusieron sus cuadros. En esta sesión se dejó a los estudiantes que analizaran fuentes de lo que es la técnica del resumen, para ello, se les sugirió utilizar algunos recursos y fuentes. Los recursos de aprendizaje, se entregaron de manera impresa y digital y estaban contemplados en la secuencia.

Sesión 4, 17 de agosto. Duración 2 horas. El elemento de competencia, fue, Comprensión de la técnica del resumen. Se revisó y comentó la tarea de la sesión anterior enfocada a la técnica del resumen, los estudiantes entregaron el resumen solicitado y comentaron. A esta actividad se le dio continuidad en esta sesión, por lo cual, dieron lectura a un material impreso, el mismo presentaba el método para elaborar el resumen, el ejemplo en dicho material para implementar esta técnica consideraba un texto relacionado con el medio ambiente. Los estudiantes de manera individual y unos en equipos, aplicaron esta técnica en un texto visto con otro docente en el curso de inducción. Los estudiantes expusieron en rotafolios y/o power point sus resúmenes. En esta sesión se dejó en un tiempo de una hora que los estudiantes realizaran y comprendieran una lectura sobre la sociología y 
la historia y que observaran dos videos sobre lo que es el cuadro comparativo. Los recursos de aprendizaje se entregaron de manera impresa y digital y estaban contemplados en la secuencia.

Sesión 5, 18 de agosto. Duración 2 horas. El elemento de competencia, fue, la comprensión del cuadro comparativo. Se revisó y comentó la tarea sobre la comprensión de un texto y de videos que hicieron referencia a los cuadros comparativos. En esta sesión y teniendo como antecedente la tarea realizada, se dio lectura y comprensión a un texto impreso relacionado con la metodología para diseñar los cuadros comparativos y la importancia de aplicarlos en algunas situaciones, una de ellas, corresponde a identificar diferencias o similitudes en textos que tienen ello por objetivo. Con referencia a la información del cuadro comparativo, los equipos ya establecidos revisaron una lectura que tenía por objetivo hacer una comparación. Los estudiantes presentaron en power point y rotafolios sus cuadros. Se dejó de tarea revisar otras técnicas y comprender los hábitos de estudio para aplicarlos en los diversos campos del saber de la licenciatura que inician. Se implementó y contestó el mismo test que se aplicó antes de elaborar el programa y tuvo como objetivo comparar los conocimientos en un antes y después de haber tomado el curso.

\section{Resultados de la evaluación del programa de técnicas y Hábitos de estudio}

Los resultados se presentan en dos secciones:

a) Segunda evaluación (Evaluación continua).

b) Tercera evaluación (Evaluación del programa).

Cabe aclarar que la primera evaluación (diagnóstica), se describe en líneas anteriores.

\section{Segunda evaluación (evaluación continua).}

La evaluación continua corresponde a la evaluación cualitativa que se realizó durante el desarrollo del curso. En el punto implementación del Programa de Técnicas y hábitos de Estudio de este documento y específicamente en los resultados de las actividades que se desarrollaron durante las cinco clases que comprendió el programa. La evaluación de los aprendizajes adquiridos se presenta a continuación:

a) En la primera sesión asistieron 22 estudiantes en el grupo " $A$ " y en el " $B$ " 27. En ambos grupos se percibió lo siguiente: 
- Comprensión del programa y la secuencia didáctica.

- Información previa deficiente sobre las técnicas y hábitos de estudio.

- Comprensión general de las técnicas y hábitos de estudio por la conferencia y los videos observados. Esto se constató por los comentarios recibidos en la plenaria.

b) En la segunda sesión asistieron 22 estudiantes en el grupo " $A$ " y en el "B" 24. En ambos grupos se percibió lo siguiente:

- Al revisar la tarea se percibió que los estudiantes comprendieron la importancia de la técnica del subrayado.

- Varias intervenciones del estudiante mostraron que comprendieron de manera reflexiva sobre el diseño e implementación de los esquemas para la comprensión de textos.

- Durante la revisión de equipos, se constató que los estudiantes diseñaban sus esquemas adecuadamente conforme se hacia la revisión del texto sugerido.

c) En la tercera sesión asistieron 22 estudiantes en el grupo "A" y en el "B" 27. En ambos grupos se percibió lo siguiente:

- Al revisar la tarea se percibió que los estudiantes diseñaron esquemas acordes a las reglas de su diseño y al exponerlos, se percibió una comprensión del tema de manera más organizada y reflexionada.

- Se percibió la comprensión del diseño de un cuadro sinóptico y en la presentación de equipos se reflejó al igual que en el esquema, que la organización de la información lleva a la comprensión y a retener aprendizajes.

d) En la cuarta sesión asistieron 23 estudiantes en el grupo "A" y en el "B" 17. En ambos grupos se percibió lo siguiente:

- Comprensión del método para elaborar un resumen.

- Comprensión de la importancia de aplicar la técnica del resumen para comprender información proveniente de materiales impresos o digitales.

e) En la quinta sesión asistieron 15 del grupo " $A$ " y 13 del "B".

- Se percibió que los estudiantes comprenden la importancia de implementar cuadros comparativos en alguna situación para comprender de manera más significativa. 
- Se constató que los estudiantes desarrollaron la habilidad para construir cuadros comparativos.

- Los resultados del test se dan a conocer en la etapa de evaluación del programa.

Durante las cinco sesiones se fueron promoviendo los hábitos de estudio y las actividades realizadas. Se constató la importancia de implementar hábitos y técnicas de estudio al observar cambios de conducta en algunos estudiantes.

La mayoría de los estudiantes contaban con información superficial de las técnicas y hábitos de estudio (los resultados de la encuesta diagnóstica (primera evaluación) se comprobaron). El saber que los conocimientos eran superficiales o aproximados, se constató en el desarrollo de las actividades. Los estudiantes contaban con habilidades mínimas para implementar estas técnicas, sin embargo, ahora, estos saberes fueron fortalecidos por los conocimientos y habilidades adquiridas con la impartición de este curso.

\section{Aplicación del instrumento en la última sesión}

De 62 estudiantes 31 contestaron el instrumento, 17 del grupo " $A$ " y 14 del grupo " $B$ ". En el grupo " $A$ " fueron 14 mujeres y 3 hombres, en el otro grupo contestaron 12 mujeres y dos hombres.

TABLA III

Concentrado del diagnóstico del nivel de conocimientos de técnicas y hábitos de estudios de estudiantes de nuevo ingreso (Grupo A y B)

\begin{tabular}{|l|c|c|c|c|c|}
\hline \multicolumn{1}{|c|}{ Variable } & 1 & 2 & 3 & 4 & 5 \\
\cline { 2 - 6 } & Nunca & $\begin{array}{c}\text { Casi } \\
\text { nunca }\end{array}$ & $\begin{array}{c}\text { Algunas } \\
\text { veces }\end{array}$ & $\begin{array}{c}\text { Casi } \\
\text { siempre }\end{array}$ & Siempre \\
\hline A.- Actitudes ante el Estudio en el Hogar & & & & & MODA \\
\hline $\begin{array}{l}\text { B.- Actitudes ante el Estudio durante la } \\
\text { Realización de la Clase }\end{array}$ & & & & & MODA \\
\hline $\begin{array}{l}\text { C.- Actitudes ante el Estudio después de la } \\
\text { Escuela }\end{array}$ & & & & & MODA \\
\hline D.- Actitudes Generales ante el Estudio & & & & & MODA \\
\hline E.- Técnicas de estudio & & & & & MODA \\
\hline
\end{tabular}

Con respecto a la tabla anterior (III), se muestran los resultados del test que se realizó en la última sesión y que fue similar a la que se aplicó en la primera evaluación. Este ejercicio, se realizó a manera de comparar los aprendizajes con los que ingre- 
saron y con los que egresaron del programa. se concentraron las respuestas de los grupos A y B, donde se aprecia que, de ambos grupos, la mayoría de los alumnos eligen como respuesta la opción 5, es decir, la MODA "Siempre". Esto denota que la mayoría de los estudiantes afirman con seguridad que ya conocen las técnicas y hábitos de estudio porque ya formaron competencias en el curso realizado.

\section{Evaluación de la eficacia del Programa de Técnicas y Hábitos de Estudio}

Para evaluar la eficiencia o ineficiencia de este programa, como se comentó en líneas anteriores, se consideró hacer una coevaluación. Esta evaluación correspondió a implementar de manera grupal preguntas abiertas sobre la eficiencia o ineficiencia del programa. Los jóvenes a quienes se les aplicó esta evaluación fueron los mismos que se mencionan en el punto anterior y las variables consideradas en las interrogantes fueron, eficacia del programa, actividades didácticas desarrolladas, material didáctico, recursos de aprendizaje y desempeño del facilitador.

De manera cualitativa, por las preguntas concernientes a las variables, eficacia del programa, la mayoría expuso que es un curso provechoso, debido a que son temas que solo se conocen, pero no se aplican y que es de gran utilidad aplicar estas herramientas en el desarrollo de sus estudios, con respecto a la variable actividades didácticas, casi todos refirieron a que fueron fáciles de desarrollar y comprender y que animaron a los aprendizajes de las técnicas y hábitos de estudio, con relación a material didáctico, casi todos comentaron que fueron adecuados, sin embargo, algunos ubicados en internet fueron difícil de conseguir y cuando se preguntó por el desempeño del facilitador que impartió el curso, lo evaluaron satisfactoriamente.

\section{Conclusiones}

Las técnicas y hábitos de estudio se asocian con el bajo rendimiento de los estudiantes. Los estudiantes de nuevo ingreso a la escuela de sociología contaban con información básica, conocimientos aproximados o previos sobre la temática, sin embargo, las competencias adquiridas por el curso implementado han fortalecido los hábitos y técnicas de estudio, ello se reflejó en los resultados que arrojaron las evaluaciones presentadas, se espera que estos saberes sean puestos en práctica para adquirir sustentablemente los aprendizajes durante el desarrollo de la licenciatura que cursaran. 
Los resultados del test que se aplicó en la primera evaluación, confirmó que, si contaban con dichas actitudes y en la última, casi todos reafirman, sin embargo, a diferencia de la primera, el resultado en la última sesión es con más certeza debido a que adquirieron aprendizajes más fortalecidos por el curso implementado. Los resultados del test fueron diferentes a los encontrados en (Acevedo, Torres y Tirado, 2015), debido a que en su investigación que analizó hábitos de estudio y motivación para el aprendizaje con estudiantes universitarios y en donde aplicó un cuestionario apegado a dicho análisis, encontró que los alumnos cuentan con poca planificación de actividades académicas, problemas emocionales, inadecuada distribución del tiempo e incorrectos métodos de estudio. Esta diferencia tal vez se da razón de que implementó identificar no solo conocimientos, sino también habilidades, en cambio, en el estudio que se presenta, interesó solamente identificar el conocimiento y la percepción que los estudiantes tenían sobre las técnicas y hábitos de estudio, no obstante, estos conocimientos fueron fortalecidos y en varios jóvenes formaron habilidades.

Los resultados de la aplicación del programa de técnicas y hábitos de estudio fueron satisfactorios ya que, a través del desarrollo de actividades realizadas, y por ende, la evaluación cualitativa aplicada durante la implementación, los tests realizados y la entrevista que se realizó al final para evaluar la eficacia de este programa arrojó que este tipo de proyectos tienen efectos positivos debido a que permiten desarrollar y promover competencias en estos campos. El programa de algún modo contribuyó en hacer conscientes a que los estudiantes sobre el uso de estas herramientas de estudio. Los resultados que se presentan en este apartado son similares a los presentados por De la Cruz y Jaimes (2012) en donde en un estudio realizado "Implementación de un proyecto de formación sobre técnicas de estudio para mejorar el rendimiento escolar de las estudiantes de tercer ciclo del Centro Escolar Eulogia Rivas de la ciudad de Cojutepeque, departamento de Cuscatlán", identificaron que con el desarrollo del programa sobre técnicas de estudio se logró el compromiso de las estudiantes de tercer ciclo en llevar a la práctica, dichas técnicas ya que son de mucha utilidad en el proceso de aprendizaje, así mismo la mayoría de docentes participaron en el programa y se comprometieron a darle seguimiento.

Finalmente, estas líneas se concluyen, haciendo énfasis en la necesidad de implementar programas educativos para fomentar las técnicas y hábitos de estudio en la universidad o en alguna institución educativa, por el hecho de que son la pieza clave para generar estrategias que coadyuven a aprendizajes sustentables para mejorar su buen rendimiento en su educación de pregrado. 


\section{BIBLIOGRAFÍA}

Acevedo, D., Torres, J. D., y Tirado, D. F. (2015). Análisis de los Hábitos de Estudio y Motivación para el Aprendizaje a Distancia en Alumnos de Ingeniería de Sistemas de la Universidad de Cartagena (Colombia). Formación Universitaria, 8(5):59.

Angulo López, E. (2011). Política Fiscal y Estratégica como Factor de Desarrollo de la Empresa Comercial de Mediano Tamaño Sinaloense, un Estudio de Caso. Tesis doctoral). México: Universidad Autónoma de Sinaloa, Facultad de Contabilidad y Administración. Culiacán, Sinaloa. Recuperado de http://www.eumed.net/ tesis-doctorales/2012/eal/seleccion muestra.html.

Arguelles, J. D. (2014). Historias de lecturas y lectores. Los caminos de los que sí leen. OCEANO Travesía. México.

Aparicio, J. L., Villaseñor, A., Casiano, I., Beltrán, J., Rodríguez, C., y Bedolla, R. (2012). Diseño, evaluación, y actualización de planes de estudio de licenciatura (En el marco del Modelo Educativo y Académico de la UAG). México: UAGro.

Barrena, J. y Molina, M. A. (2010). Didáctica de técnicas de estudio y desarrollo emocional. Trances, 3(1):156.

Castro, M. (2005). La investigación del entorno natural. Una estrategia didáctica para la enseñanza-aprendizaje de las ciencias naturales. Universidad Pedagógica Nacional. Colombia. p. 33.

Castillo Rojas, S. V. (2015). Hábitos de estudio en estudiantes de bibliotecología, Universidad Nacional Autónoma de México. Códices, 11(2), 126.

Cambrón, J. E., Chávez, J. K. y Llamas, D. U. (2016). Dónde, cuándo y cómo estudiar: la vigencia del diagnóstico sobre técnicas y hábitos de estudio en universitarios. 2:157.

Casaña, G. S. (2015). Competencias didácticas para la educación ambiental. Revista Vinculando. http://vinculando.org/educacion/competencias-didacticaseducacion-ambiental.html

Colegio-Peter-College. Test para evaluación de hábitos de estudios, disponible en: https:// webcache.googleusercontent.com/search?q=cache:wZjA2b3bYFQJ:https:// erikamunoz.files.wordpress.com/2007/07/test-para-evaluacion-de-habitos-deestudios. $\mathrm{doc}+\& \mathrm{~cd}=1 \& \mathrm{ch} \mid=\mathrm{es} \& \mathrm{ct}=\mathrm{c} \operatorname{lnk\& gl=mx}$.

DefinicionABC Tu diccionario hecho fácil. Definición de Técnica de Estudio. Disponible en: https://www.definicionabc.com/comunicacion/tecnicas-de-estudio.php.

De la Cruz, C. M., Jaimes, S. E. (2012). “Implementación de técnicas de estudio para mejorar el rendimiento escolar de las estudiantes de tercer ciclo del centro escolar Eulogia Rivas de la Ciudad de Cojutepeque, departamento de Cuscatlan, durante el periodo de octubre 2011 a octubre 2012. Trabajo de graduación preparado para la Facultad Multidisciplinaria Paracentral. San Vicente. Universidad de el Salvador. 1,115. Consultado en: http://ri.ues.edu.sv/2282/1/entrega de tesis 3.pdf

Enríquez, M. F. (2013). Hábitos y técnicas de estudio en la Universidad Mariana. Revista UNIMAR, 31(2), p. 89. 
Elizondo, I. (2000). Propuesta para planear estrategias didácticas en el proceso enseñanzaaprendizaje. Tesis para obtener el grado de maestría en la enseñanza de las ciencias con especialidad en Biología. Universidad Autónoma de Nuevo León. p.22.

Galagovsky, L. R. (2004). Del aprendizaje significativo al aprendizaje sustentable. Parte 2: Derivaciones comunicacionales y didácticas. 22(3), p.350.

García, K. y Alviarez, T. A. (2011). Estrategias para el para el aprendizaje significativo y su relación con el rendimiento académico en inglés. Synergies Venezuela, 6.

Gutiérrez, S. y Montañez, G. S. (2012). Análisis teórico sobre el concepto de rendimiento escolar y la influencia de factores socioculturales. Revista Iberoamericana para la Investigación y el Desarrollo Educativo, 9: 1-21.

Guerrero, M. P. S. (2014). Estilos de aprendizaje y rendimiento académico en alumnos que cursaron genética clínica en el periodo de primavera 2009 en la facultad de medicina de la benemérita universidad autónoma de puebla. Revista Estilos de Aprendizaje 5(5): 1-14.

Guirao, P. (2013). Técnicas y hábitos de estudio de la asignatura de historia en secundaria y bachillerato. GeoGraphos, 4(42), 242.

Marcelo, Ch. (2011). Técnicas de Estudio y Rendimiento Académico. Rev Inv Sci, 1(1).

Modelo Educativo de la Universidad Autónoma de Guerrero (2013), p.31.

Navarro, E. (2003). El rendimiento académico: concepto, investigación y desarrollo". Revista Electrónica Iberoamericana sobre Calidad, Eficacia y Cambio en Educación. 2:1-15.

Publicaciones Vértice S.L. (2008). Tánicas de Estudio. Edit. AENOR ER. España. P. 5.

Rodríguez, L. (2014). Metodologías de enseñanza para un aprendizaje significativo de la Histología. Rev. Dig. Universitaria, 15(11), 1.

Sebastián, A., Ballesteros, B., y Sánchez, M. (2012). Universidad Nacional de Educación a Distancia. Técnicas de estudio. p. 3. http://qinnova.uned.es/archivos publicos/ qweb paginas/3439/tecnicasdeestudio.pdf

Serrano González-Tejero, J. M., y Pons Parra, R. M. (2011). El constructivismo hoy: enfoques constructivistas en educación. Revista electrónica de investigación educativa, 13(1), 1-27..

SEP. Secretaría de Educación Pública (2014). Manual para impulsar mejores hábitos de estudio en Planteles de Educación Media Superior. 7,11.

SEP. Modelo Educativo 2016. El Planteamiento Pedagógico de la Reforma Educativa. 36-46.

Sepúlveda, G. y Lucia, I. (2017). Técnicas de Estudio. PublicacionesDidácticas.com, 79, 420, 422.

UAGro. Anuario Estadístico 2013-2014 de la Universidad Autónoma de Guerrero.

UAGro. Anuario Estadístico 2014-2015 de la Universidad Autónoma de Guerrero. p. 47-49.

Valero, M.C. (2011). Técnicas de Estudio. Revista de Clases historia. 229, 2. 
